\title{
Téoros
}

Revue de recherche en tourisme

\section{Le produit touristique montréalais : à l'heure de la transition}

\section{Pierre Bellerose}

Volume 10, numéro 3, novembre 1991

Destination Montréal : d’hier à demain

URI : https://id.erudit.org/iderudit/1079194ar

DOI : https://doi.org/10.7202/1079194ar

Aller au sommaire du numéro

Éditeur(s)

Université du Québec à Montréal

ISSN

0712-8657 (imprimé)

1923-2705 (numérique)

Découvrir la revue

Citer cet article

Bellerose, P. (1991). Le produit touristique montréalais : à l'heure de la transition. Téoros, 10(3), 3-5. https://doi.org/10.7202/1079194ar d'utilisation que vous pouvez consulter en ligne.

https://apropos.erudit.org/fr/usagers/politique-dutilisation/ 


\section{Le produit touristique montréalais: à l'heure de la transition}

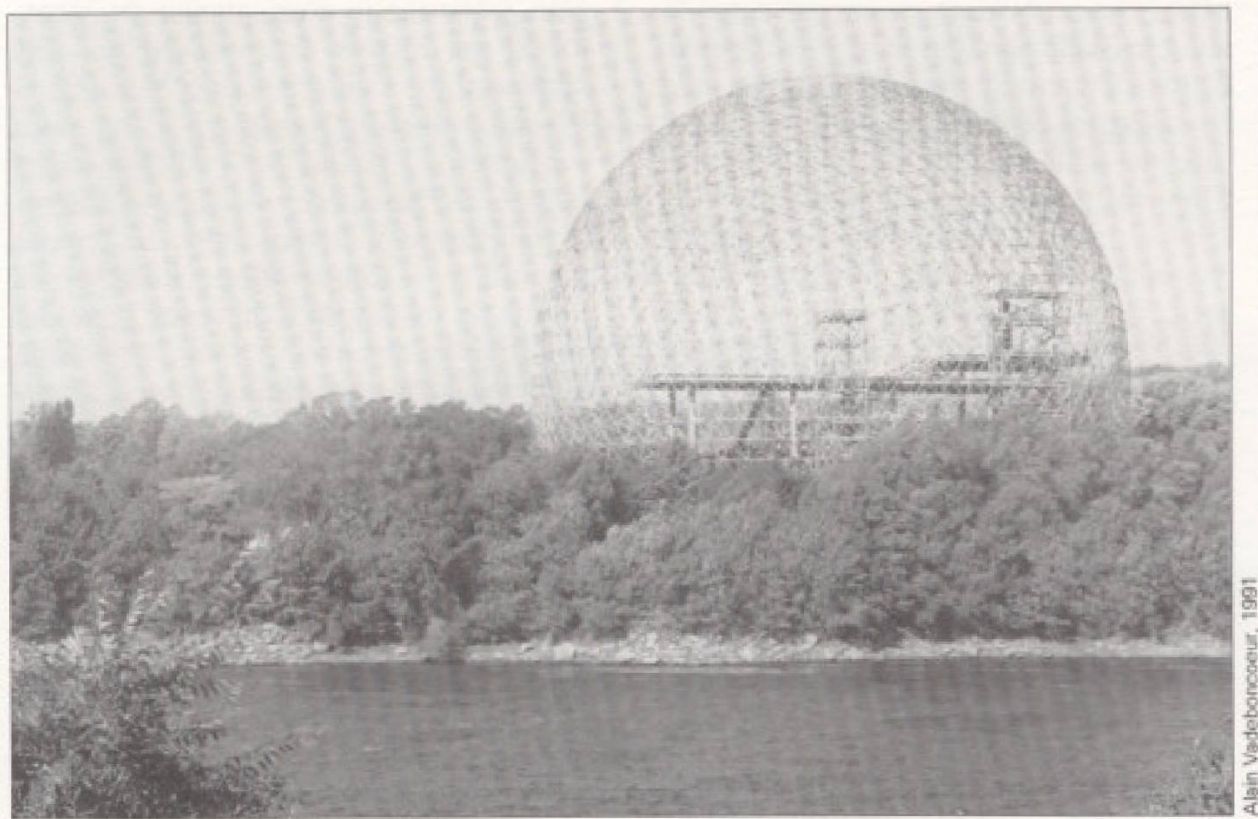

La Biosphère, dans le Pare des Iles, connaitra prochainement une cure importante de rajeunissement en accueillant le centre d'interprétation et de protection de l'environnement.

\section{0-1976: ère des grands projets pour Montréal}

Montréal a connu sa première et principale période de développement intensif de ces institutions culturelles et touristiques entre 1960 et 1976 . Les équipements apparus précédemment (ex.: Basilique Notre-Dame (1829), Musée des BeauxArts de Montréal (1860), Musée McCord (1882), l'Oratoire St-Joseph (1955), le Jardin botanique (1947), etc.) s'implantèrent de façon discontinue dans le temps.

En effet, la période 1960-1976 fut marquée par une série d'aménagements et de réalisations à caractère touristique qui façonnent encore laréalité montréalaise d'aujourd'hui, soit les aménagements des îles Ste-Hélène et Notre-Dame dans le cadre de l'Expo '67, les premières ententes entre le ministère des Affaires culturelles et la Ville de Montréal qui permirent des travaux importants de rénovation et de mise en valeur du Vieux-Montréal, l'ouverture du métro

Monsieur Pierre Bellerose et directeur de la recherche et du developpement a I'Office des congress et du tourisme du Grand Montreal (OCTGM).
(1966) et le développement du réseau piétonnier intérieur, l'ouverture du Musće d'Art Contemporain (1961) et son installation à la Cité du Havre (1967), l'Aquarium de Montréal (1966), le Planétarium de Montréal (1966), le deuxième agrandissement du Musće des Beaux-Arts (1976) et l'ouverture du Stade olympique sans son mât (1976).

Cette effervescence était liée à un certain rattrapage au niveau des infrastructures culturelles et touristiques mais aussi et surtout à cause des grands événements que furent l'Exposition universelle de 1967 et les Jeux olympiques de 1976. Parallèlement à cela, le tourisme d'affaires, grâce à l'ouverture de grands hôtels axés sur les congrès (ex.: Reine Elizabeth et Château Champlain) et de la Place Bonaventure en 1967, connut aussi une croissance non négligeable.

\section{7-1986: I'ère des événements}

Malheureusement, la décennie qui succéda aux Jeux olympiques fut marqué par un niveau très bas des investissements privés et publics dans les équipements récréotouristiques. La seule exception notable, le Palais des congrès, ouvrit ses portes en 1983 mais ne prit son réel envol qu'à la fin des années 1980.

Trois raisons peuvent expliquer ce vieillissement des infrastructures touristiques. Dans un premier temps, la dette astronomique liée aux Jeux olympiques amenèrent sans doute le gouvernement québécois à prioriser d'autres types de dépenses. Deuxièmement, la récession de 1981-1982 força les différents gouvernements à reporter plusieurs projets et finalement, force est d'admettre que les années 1970 furent caractérisées par un penchant favorable pour le régionalisme, ce quin'aida pas la situation de Montréal.

D'ailleurs, le portrait que faisait les analystes du produit touristique au milieu des années 1980 était plutôt sombre. Ainsi, selon l'Office de planification et de développementdu Québec, leséléments attractifs de Montréal étaient composés «d'une variété et une abondance d'attraits physiques concentrés dans cinq zones: 1) l'île Ste-Hélène et ses abords, le Vieux-Montréal, le centreville, le Mont-Royal et le Parc olympique. Cette abondance est toutefois très relative, car plusieurs attraits secondaires attirent peu les touristes qui, durant leur court séjour à Montréal, visitent principalement Terre des Hommes, l'Oratoire Saint-Joseph, le Jardin botanique et la Basilique Notre-Dames.

«De plus, il y a une absence totale d'intégration entre les attraits situés à l'intérieur d'une zone ainsi qu'entre les quatre zones touristiques centrales. Cellesci ne présentent d'ailleurs pas une image touristique très reluisante. Les îles de Terre des Hommes sont à la recherche d'une nouvelle vocation, les vestiges de l'Expo ayant perdu leur pouvoir d'attraction. La mise en valeur du Vieux-Montréal est fort inégale et la revitalisation amorcée depuis plus de vingt ans s'avère une entreprise laborieuse. Le centre-ville est le lieu privilégié des images contrastantes et disgracieuses: les parkings, les immeubles victo- 
riens et les complexes commerciaux s'entremêlent sans harmonic, donnent à Montréal l'image d'une ville "bombardée" $x^{(1)}$

Jusqu'à tout récemment, Montréal possédait très peu d'équipements jugés excellents ou valant le voyage, si on tient compte des critères de la plupart des guides (dans le cas du guide Michelin, seul le complexe olympique méritait trois étoiles).

En partie pour compenser le peu d'équipements permanents offerts aux visiteurs, les décideurs publics du secteur touristique ont appuyé d'une façon importante les festivals de portée internationale qui ont vu le jourà partir de la fin des années 1970 tels le Festival des Films du Monde, le Grand Prix Molson du Canada de course automobile, le Festival International de Jazz de Montréal et le Festival Juste pour Rire. Ce phénomène a eu pour effet de créer une nouvelle image de dynamisme de Montréal mais aussi d'entraîner une intensification des activités offertes sur une période d'à peine deux mois pour retomber un peu à vide le reste de l'année.

\section{7-1993: I'ère des équipements}

Tout en conservant les festivals qui ont fait la marque de Montréal, on assiste depuis 1987 à une seconde période d'effervescence importante en ce qui concerne les équipements de Montréal qui peuvent servir le produit touristique. Ces investissements, en majorité de nature publique, ont lacaractéristique de venir consolider les cinq pôles touristiques de Montréal. Voici une brève description des investissements dans chacun des pôles.

\section{Le Vieux-Montréal}

Le Vieux-Montréal, qui avait connu de nombreux travaux de rénovation durant les années 1960, a vécu une période plutôt difficile par la suite jusqu'au milieu des années 1980. Toutefois, on constate, pour la période allant de 1987 à 1993, des investissements privés et publics de près d'un milliard de dollars ${ }^{(2)}$ dont le Vieux-Port, le Centre de Commerce Mondial et l'hôtel Intercontinental, la rénovation du Centre d'histoire de Montréal, le Centre d'interprétation de la Pointe-à-Callières (ouverture en 1992) et la réfection de la rue de la Commune (actuellement en cours).
Ce renouveau est lié étroitement au leadership de la SIMPA qui a su coordonner les investissements des intervenants privés et ceux du secteur public.

\section{Le Pôle Maisonneuve}

Malgré les problèmes que connaît actuellement le Stade olympique, le Pôle Maisonneuve s'impose de plus en plus et en vient à rivaliser avec le Vieux-Montréal pour le titre du pôle attractif par excellence de Montréal. Contrairement au Vieux-Montréal, le positionnement touristique du Pôle Maisonneuve est récent. Il débuta en fait avec la finalisation du mât du Stade olympique qui a attiré plus de 400000 visiteurs (au funiculaire) en 1988. Par la suite, le dynamisme se manifesta à travers le Jardin botanique grâce à la création du Jardin japonais (1989), de l'Insectarium (1990) et du Jardin chinois (1991) qui font maintenant de cette institution une attraction unique en Amérique du Nord. Finalement, le projet innovateur du Biodôme assurera le potentiel attentif de ce secteur et la croissance de la clientele.

\section{Le centre-ville}

Le renouveau touristique du centre-ville se manifeste à deux niveaux. Dans un premier temps, les projets de bonification du réseau muséologique de Montréal se concrétiserontaprès souvent plus de dix ans d'attente. On pense principalement aux projets d'agrandissements du Musée des BeauxArts et du Musée McCord et à la relocalisation du Musée d'Art Contemporain qui viendra ainsi consolider la Place des Arts. Ces projets d'agrandissements viennent $s$ 'ajouter au magnifique Centre canadien d'architecture qui a ouvert ses portes en 1989.

D'autre part, depuis quelques années, la trame commerciale du centre-ville s'est modifiée considérablement avec l'apparition de nouvelles galeries commerciales telles les Cours Mont-Royal (1988), les Promenades de la Cathédrale (1990), la Place Montréal Trust (1989) et le Centre Eaton (1990). Cela a eu aussi pour effet d'accentuer l'expansion et l'utilisation du réseau piétonnier intérieur de Montréal, On doit aussi souligner l'avenue McGill College qui a êtế redessinée récemment afin d'en faire ressortir les édifices qui la longent. Toutefois, beaucoup de travail reste encore à faire comme en fait foi les espaces vacants sur le pourtour de la Place des Arts.

\section{Le Mont-Royal/Oratoire}

La Ville de Montréal a déposé en 1990 un plan préliminaire d'orientation du MontRoyal qui mettait en évidence les trois sommets que l'on retrouve à Montréal, à Westmount et à Outremont. Une vaste consultation a eu lieu en 1990 et on attend le dépôt du Plan final d'orientation du MontRoyal pour le printemps 1992. La priorité sera mis sur la conservation et les acquis au niveau du patrimoine bâti (entre autres en ce qui concerne les belvédères). La Ville de Montréal s'est engagée à dépenser 9 millions de dollars sur trois ans à ce chapitre.

\section{Le Parc des illes}

Comme le souligne Pierre Labrie dans son article sur les retombées du 350 e anniversaire sur le tourisme à Montréal (voir à l'intérieur de ce numéro), le gouvernement fédéral investit dans un premier temps 55 millions de dollars pour l'aménagement d'un nouveau parc urbain dans la partie ouest de l'île Ste-Hélène (comprenant une aire de spectacle en plein air, un lac et un promontoir) et dans un deuxième temps, 17.5 millions de dollars dans la restauration de la Biosphère et de l'aménagement d'un Centre d'interprétation et de protection de l'environnement (ouverture en 1993).

\section{L'après 1992}

Malgré le redressement que l'on connaît actuellement, Montréal aura, dans l'avenir, encore beaucoup de difficultés à se positionner devant la concurrence grandissante. Bien sûr, Montréal n'aura jamais le niveau d'attractivité de New York, Paris ou Londres mais on ne doit pas pour autant se laisser devancer par des villes comme Boston, Washington, Toronto, San Francisco ou New Orleans qui n'ont jamais arrêté d'améliorer le niveau de compétitivité de leur destination.

Il n'y a pas eu jusqu'ici d'exercice formel de réflexion visant à l'élaboration d'une vision stratégique du développement du produit touristique montréalais et ainsi préparer l'après 1992. Toutefois, certains constats permettent de proposer deux axes de 
développement pour le produit touristique d'ici l'an 2000 soit la complétion de la trame muséologiqueetle tourisme d'affaires et de congrès.

\section{La trame muséologique}

Montréal, en tenant compte des ajouts à venir en 1992, possède une bonne trame muséologique avec entre autres le Centre canadien d'architecture, le Musée McCord, le Musće des Beaux-Arts, le Musée d'Art Contemporain et le futur Musée de l'humour, le Lieu historique national du commerce de la fourrure, le Château Ramesay, etc.

Cependant, dans la perspective du produit touristique montréalais en l'an 2000, il est essentiel de continuer d'améliorer notre trame musćologique par l'ajout de quelques muséesqui viendrontcompléter l'offre déjà existante. En fonction de l'information que nous possédons et de l'état d'avancement actuel de certains dossiers, il me semble pertinent de présenterà titre indicatif quatre (4) propositions prioritaires.

- Musée de la science et de la technologie

Pour l'éducation scientifique de la population en général comme pour le tourisme, un musée de la science et de la technologie entraîne un très grand achalandage. On n'a qu'à penserà l'Ontario Science Center. Cet équipement devrait être localisé soit au Vieux-Port soit à l'intérieur du Pôle Maisonneuve pour créer une concentration majeure propice au tourisme familial.

\section{- Musée de l'enfance}

Devant le succès remporté à Boston par un tel musée, il nous apparaît important d'ajouter cet équipement, au Vieux-Port, comme le recommande les études préliminaires qui ont été faites sur ce dossier.

Lesecteurdu tourisme familial nousapparait très important et il existe un besoin d'offrir plus d'activités pour les enfants. Une étude réalisée par SECOR en 1988 constatait qu'une des principales faiblesses du produit touristique montréalais était le manque d'activités pour les enfants ${ }^{(3)}$.

\section{- Musée des arts décoratifs}

Grâce à la Fondation MacDonald Stewart, Montréal possède la plus importante col- lection enart décoratif de lapériode d'aprèsguerre en Amérique. Le musée, logé au château Dufresne, ne répond pas aux normes muséologiqueset procure très peud'espace d'exposition. La Fondation cherche à relocaliser ce musée. Il s'agirait là d'un musée au contenu original par rapport à ce que l'on retrouve dans les destinations concurrentes.

\section{- Musée de l'image}

La Cinémathèque québécoise est maittre d'oeuvre du musée de l'image en mouvement (cinéma, télévision et vidéo). Ce musée serait localisé à la Cinémathèque, soit Maisonneuve et Saint-Denis. Cemusée aurait l'avantage de renforcer un segment important du secteur des industries culturelles montréalaises.

\section{Le tourisme d'affaires et de congrès}

Montréal a aussi une autre vocation qui est celle d'une destination pour le tourisme d'affaires et de congrès. Il faut noter que ce secteur a connu une hausse de $30 \%$ au cours des dix dernières années au Canada, alors que le tourisme d'agrément stagnait durant la même période ${ }^{(4)}$. Par ailleurs, Montréal est la première ville au Canada pour les congrès internationaux et la troisième en Amérique après New York et Washington.

Il faut dès maintenant décider de l'agrandissement du Palais des congrès, à la fois pour pouvoir attirer plus de congrès et solliciter des congrès regroupant plus de 10000 délégués. Il s'agit là d'une condition sine qua non si nous voulons maintenir cet acquis de destination de congrès internationaux et tirer notre épingle du jeu sur le marché après 1995. La mise en place d'un casino au Palais des congrès serait d'ailleurs un atout supplémentaire à la fois pour le tourisme de congrès mais aussi pour le tourisme d'agrément.

Il est également nécessaire d'ajouter un espace pour les salons, foires et expositions d'au moins $200000 \mathrm{pi}^{2}$, idéalement rattaché au Palais des congrès. Les espaces actuels (Place Bonaventure, Palais, Stade) ne peuvent accepter aucune autre activité à leur calendrier (voir à ce propos l'article de François Cadoret dans ce numéro).

Par ailleurs, Montreal est la seule ville canadienne où se retrouve le siège d'un organisme des Nations-Unies, l'OACI.
D'autres organismes internationaux tels 1'IATA, le Secrétariat mondial pour la protection de la couche d'ozone, etc., ont pignon sur rue à Montréal. Tant pour la réputation internationale de Montréal, ce qui rejaillit sur notre renommée de destination touristique importante, que pour le nombre de réunion d'experts, assemblées, visites techniques, ce qui augmente l'achalandage, il est important de consolider le rôle international de Montréal. C'est pourquoi la réalisation du projet de Centre international de conférences est une priorité dans la perspective du développement du produit touristique.

\section{Conclusion}

Malgrế le nombre important de nouveaux équipements en construction actuellement a Montreal, un consensus semble se dessiner au sein du milieu touristique: la période de rattrapage étant sur le point de se terminer, il faut rapidement préparer l'après 1992 et ainsi Eviter une longue période de stagnation comme celle que l'on a vécu entre le milieu des années 1970 et le milieu des années 1980. Il existe présentement des projets sur la table fort intéressants et une clientèle qui attend ces nouvelles infrastructures (entre autres au niveau des congrès et des foires commerciales). La période de transition étant sur le point de se terminer, Montréal estàl'heure du choix de sesprioritéspour assurer son développement touristique d'ici l'an 2000.

\section{Notes et références}

(1) OPDO, Le tourisme à Montreal: bilan et perspectives de développement, 1986, p. 5 .

[2] aDéveloppements touristiques et immobiliers du Vieux-Montréabr, in Le projet Vieux-Montrèal, vol. 3, no 1 , juin 1990, p. 1 .

(3) SECOR, Étudedu comportement et del'attitud des différentes clientéles touristiques à Montráal, $1989,0.74$.

(4) Statistique Canada, Enquète des voyages des canadlens. 